\title{
Itching Gingiva: An Early Sign of Pre-Inflammatory Pulp Necrosis or Unsuccessful Endodontic Treatment - A Case Series
}

\author{
${ }^{1}$ Department of Fixed Prosthodontics, Damascus University, Syria \\ ${ }^{2}$ Department of Oral Medicine, Syrian Private University, Syria \\ ${ }^{3}$ King's College London Dental Institute, Denmark Hill Campus, UK \\ ${ }^{4}$ Eastman Dental Institute, University College London, UK
}

Fendi Alshaarani ${ }^{1}$, Imad Barngkgei ${ }^{2 *}$, Easter Joury ${ }^{3}$ and Kanaan Elias ${ }^{4}$

*Corresponding author: Dr. Imad Barngkgei, DDS, MSc, PhD, Department of Oral Medicine, Faculty of Dentistry, Syrian Private University, Damascus, Syria, Tel: 00963-932785671

\begin{abstract}
Pulp necrosis or unsuccessful endodontic treatment may be a painful process or be completely asymptomatic, regardless of their radiographic features. However, there is no report in the literature regarding the presence of an early sign of pulp necrosis preceding pulpal pain, incomplete endodontic fillings or radiographically complete endodontic fillings performed under unsterilized conditions. In this case series, we described four cases who had a common complaint of itching gingiva. The gingival itching, from which they suffered, disappeared after performing/redoing the endodontic treatment. Thus, itching gingiva may be an early sign of pre-inflammatory pulp necrosis or unsuccessful endodontic treatment.
\end{abstract}

\section{Keywords}

Endodontics, Itch, Pulp necrosis

\section{Key Messages}

Itching gingiva may be an early sign of pre-inflammatory pulp necrosis or unsuccessful endodontic treatment.

\section{Introduction}

The ultimate aim of endodontic therapy is to preserve the involved teeth healthy and functional in the oral cavity. Success rates of endodontic treatment is up to $86-98 \%$ [1]. Unsuccessful endodontic treatments are characterized by presence of clinical symptoms, continuous of preexisted symptoms, appearance of periapical lesion (if not previously existed), remaining or increas- ing in size of already existed lesion, occurrence of root resorption, or continuous of preexisted resorption [2].

Anyhow, pain is the main symptom associated with pulpal injuries or post failure endodontic treatments. Nevertheless, it is not uncommon that pulp necrosis occurs with little or no pain [3-5]. There is no report in the literature regarding the presence of an early sign of pulp necrosis preceding pulpal pain, incomplete endodontic fillings or radiographically complete endodontic fillings performed under unsterilized conditions.

Itch is the dominant symptom of a vast variety of dermal diseases, which has been found to affect the quality of life of affected person [6].

Itch was classified into four different types: Neurogenic (result from disorders that affect organ systems other than the skin), psychogenic (associated with psychological abnormalities), neuropathic (results from damage to central or peripheral sensory neurons), and pruritoceptive (generated in the skin either through inflammation or skin damage) $[7,8]$.

The thick myelinated $A \beta$ fibers transmit tactile sensation, whereas the thinly myelinated $A \delta$ and unmyelinated C-fibers are mainly involved in the conduction of thermal and pain/itch sensation [9]. In the oral cavity, itch is an extremely rare symptom and most commonly found in infants when teeth start to erupt [10].

Citation: Alshaarani F, Barngkgei I, Joury E, Elias K (2018) Itching Gingiva: An Early Sign of Pre-Inflammatory Pulp Necrosis or Unsuccessful Endodontic Treatment - A Case Series. Int J Oral Dent Health 4:063. doi.org/10.23937/2469-5734/1510063

Accepted: October 10, 2018: Published: October 12, 2018

Copyright: (c) 2018 Alshaarani F, et al. This is an open-access article distributed under the terms of the Creative Commons Attribution License, which permits unrestricted use, distribution, and reproduction in any medium, provided the original author and source are credited. 

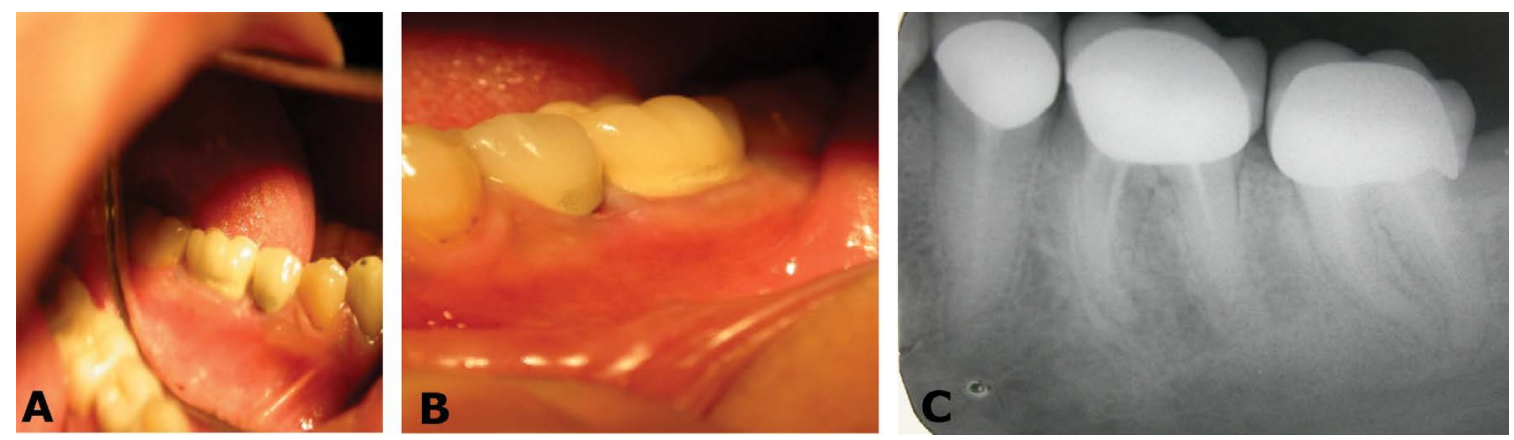

Figure 1: A) Clinical pictures of the gingival area of 46 in case number 2; B) The slightly red appearance of the gingiva is due to rubbing with patient's nail; C) A periapical radiography showing unsuccessful endodontic treatment of 36 .

In this case series, we described four cases for three female and one male patients, which had a common complaint of itching gingiva. They were referred to our department for further investigations. History revealed that all these four patients were worried that the gingival itching might be an early sign of a serious disease or malignant tumor. They consulted a number of dentists who assured them the absence of any abnormal findings upon clinical and radiographic examinations. However, their concerns and anxiety continued because the gingival itching had been persistent. The reassurance given to them by the consulted dentists did not resolve their anxiety or addressed their concerns. The oral hygiene of these patients was very good.

\section{Case Number 1}

A 50-years-old man suffered from gingival itching in the left mandibular first molar (36) area, though the gingiva appeared healthy upon the clinical examination. Patient's oral hygiene was good. The symptoms started two weeks before his visit to the dental clinic. As the gingival itching caused him uncomfortable sensation and anxiety, he used hard toothbrush and toothpicks to clean that area. However, gingival itching persisted.

The patient was referred to a periodontist, whose diagnosis was normal gingival tissue. Chlorhexidine mouth rinse was prescribed. No improvement was observed. The periapical radiograph revealed no periapical lesions. The tooth had received a fixed metal crown, which was de-cemented and removed about one year before patient's visit to our department. Thermal vitality pulp test (using ethyl chloride in a spray) revealed pulp necrosis. Endodontic treatment was performed, and the tooth was restored with amalgam. Gingival itching disappeared. The tooth was crowned one month later.

\section{Case Number 2}

A 40-years-old woman was referred to our department with gingival itching in the area of the right mandibular first molar (46). The symptoms started 6 months after the endodontic treatment of this tooth. The patient had no pain, but there was an uncomfortable sensation in that tooth's area. The gingiva appeared healthy during the clinical examination (Figure $1 \mathrm{~A}$ and Figure 1B).
The periapical radiograph showed an incomplete endodontic filling of the distal root (Figure 1C). After endodontic retreatment of this tooth, the symptoms disappeared within two days.

\section{Case Number 3}

A 19-years-old woman with a fixed bridge extended from the right maxillary lateral incisor (12) to the left maxillary central incisor (21). The right maxillary central incisor (11) was extracted. Both abutments (12 and 21) were endodontically treated. The patient was referred to us, because she suffered from tingling and itching in the upper lip and gingiva in the area of the lateral incisor. The symptoms started when the bridge was cemented. The gingiva was healthy as per the clinical examination. The endodontic filling of the tooth was radiographically acceptable.

The bridge was removed, and the endodontic treatment was redone. It was noticed that the previous endodontic treatment was not carried out under ideal conditions of sterilization. Periapical curettage was performed surgically. The symptoms disappeared. A new fixed bridge was constructed and cemented.

\section{Case Number 4}

A 42-years-old woman was referred to our department by her dentist. A fixed bridge extended from the right maxillary second molar (17) to the left maxillary canine (23). The symptoms started three days following the endodontic therapy of 21. The endodontic filling of this tooth was $2 \mathrm{~mm}$ deficient (from the apex) as per the periapical radiograph. The endodontic therapy was redone without removing the bridge. It was also noticed that the previous endodontic treatment was not carried out under ideal conditions of sterilization. The symptoms disappeared after redoing the endodontic treatment.

\section{Discussion}

The clinical success of endodontic treatments is characterized by the absence of any clinical signs or symptoms. Otherwise, endodontic treatment should be considered as failure [11]. However, Friedman and Mor introduced the term functional tooth as a third catego- 
ry in the debate of success/failure of endodontic treatment [12]. It means that a functional tooth may include the presence of apical periodontitis, when the patient is asymptomatic. Bender, et al. [13] recommended, based on radiographic and histological findings, that lesions which do not change in size should be considered acceptable.

Before deciding to retreat, it is important to use a long enough observation period after treatment has been completed. Healing of periapical tissues is a dynamic process and it is possible that a premature evaluation of periapical healing may occur if the repair process has not yet stabilized. Signs and symptoms of infection should be analyzed as well [14]. Endodontic treatment evaluation may be performed clinically, radiographically and microscopically, although the later cannot be done except after the extraction of the involved tooth or when apicectomy is performed [15].

Recently, some authors advocated the use of CBCT for follow up purposes after endodontic treatment. This is because CBCT has the ability to detect apical radiolucency with higher sensitivity than intraoral radiographs [16]. Should we start using CBCT in cases with unsuccessful endodontic treatments or when success is questionable? Could CBCT have been detected unusual findings if we had used it in our cases?

Clinical features of endodontic failure include; Sensitivity, discomfort and/or pain, edema, fistula, excessive mobility. Radiographic features of endodontic failure include periapical bone radiolucency and increasing in size of pre-existed radiolucency $[11,14,15]$. On the other hand, clinical follow-up studies on endodontic therapy are rare, most of which are those applied the modern endodontic techniques [17]. Modern techniques provided better outcome than hand instruments [18]. Hence, the few numbers of follow up studies which basically performed with modern techniques may explain why itching gingiva was not noticed as an early sign of necrotic or unsuccessful endodontic treatment.

A mediator of itch (a pruritogen) can be defined as a substance that elicits both the sensation of itch and an urge to scratch. These mediators interact with molecular detectors, which detect and respond to a diverse range of ligands or stimuli. Itch is thus driven by a variety of mediators that interact with detectors present on the surface of nerve fibers.

Conventionally, histamine and serotonin had been the paradigm for in vivo modeling of pruritoceptive itch, but other substances such as prostaglandins, neuropeptides, interleukin-31, and proteases have been involved in the itch field.

In addition, bacteria and bacterial products reach the periapical area in cases with pulp necrosis or unsuccessful endodontic treatments. Acute or chronic inflammatory response is formed. Immuno-cells in the chronic periapical inflammation produce a number of chemicals necessary for the inflammatory process. These chemicals include histamine and serotonin; Which are responsible for hypersensitivity reactions $[19,20]$. Furthermore, both interleukin-31 and prostaglandins are produced during the inflammatory process. As a result, the periapical area of failed endodontically-treated teeth or teeth with necrotic pulp contains many substances that may induce itch in the nerve in the periapical area. Perhaps, the inflammatory process is not so acute to produce pain.

Accordingly, some of the histamine and serotonin produced in the inflamed area may reach the gingiva. Alternatively, due to the fact that the gingiva has the same sensory branches of the nearby tooth, perhaps the itch sense (originated from the periapical area) may be felt as it were originated from the nearby gingiva. This may explain the reasons for behind the gingival itching among the cases of the current report.

If the root filling looks radiographically unsatisfactory but is successful, then it would be beneficial to further monitor this given that the quality of shaping and obturation of the canals are not the only determining features of endodontic success, though they may be the only features presented on an endodontic radiograph [14]. This was confirmed in other studies, which found that in teeth without cultivable bacteria at the time of filling, the root filing status, whether filled adequately or not, had no impact on the outcome [21,22]. The later however cannot be evaluated routinely in the clinical practice.

The abovementioned cases showed that gingival itching around an endodontically-treated tooth may be a symptom of pulp necrosis or unsuccessful endodontic therapy. Further studies are highly needed to validate this finding, which might be an important symptom for decision-making regarding the need for redoing the endodontic therapy. In conclusion, itching gingiva may be an early sign of pre-inflammatory pulp necrosis or unsuccessful endodontic treatment as shown in the four cases included in the present report.

\section{References}

1. Song M, HC Kim, W Lee, E Kim (2011) Analysis of the cause of failure in nonsurgical endodontic treatment by microscopic inspection during endodontic microsurgery. $\mathrm{J}$ Endod 37: 1516-1519.

2. European society of endodontology (2006) Quality guidelines for endodontic treatment: Consensus report of the european society of endodontology. Int Endod J 39: 921-930.

3. Woda A, Ddoméjean-Orliaguet S, Faulks D, Augusto F, Boudeau L, et al. (1999) Réflexions sur les critères diagnostiques des maladies pulpaires et parodontalesd'origine pulpaire. Inf Dent 43: 3473-3478.

4. Bergenholtz G (2000) Evidence for bacterial causation of adverse pulpal responses in resin-based dental restorations. Crit Rev Oral Biol Med 11: 467-480. 
5. Cai S, W Zhang, G Tribble, W Chen (2017) Reactions of human dental pulp cells to capping agents in the presence or absence of bacterial exposure. J Oral Sci 59: 621-627.

6. Kini SP, LK DeLong, E Veledar, AM McKenzie-Brown, M Schaufele, et al. (2011) The impact of pruritus on quality of life: The skin equivalent of pain. Arch Dermatol 147: 11531156.

7. Lilit Garibyan, Curtis G Rheingold, Ethan A Lerner (2013) Understanding the pathophysiology of itch. Dermatol Ther 26: 84-91.

8. Yosipovitch G, Greaves MW, Schmelz M (2003) Itch. Lancet 361: 690-694.

9. Lawson SN (2002) Phenotype and function of somatic primary afferent nociceptive neurones with c-, adelta- or aalpha/beta-fibres. Exp Physiol 87: 239-244.

10. Feldens CA, Faraco IM, Ottoni AB, Feldens EG, Vitolo MR (2010) Teething symptoms in the first year of life and associated factors: A cohort study. J Clin Pediatr Dent 34: 201-206.

11. Tabassum S, Khan FR (2016) Failure of endodontic treatment: The usual suspects. Eur J Dent 10: 144-147.

12. Friedman S, Mor C (2004) The success of endodontic therapy-healing and functionality. J Calif Dent Assoc 32: 493503.

13. Bender IB, Seltzer S, Soltanoff W (1966) Endodontic success-a reappraisal of criteria II. Oral Surg Oral Med Oral Pathol 22: 790-802.
14. Hansrani $V$ (2015) Assessing root canal fillings on a radiograph-an overview. Br Dent J 219: 481-483.

15. Estrela C, Holland R, Estrela CR, Alencar AH, Sousa-Neto MD, et al. (2014) Characterization of successful root canal treatment. Braz Dent J 25: 3-11.

16. Lofthag-Hansen S, Huumonen S, Grondahl K, Grondahl HG (2007) Limited cone-beam CT and intraoral radiography for the diagnosis of periapical pathology. Oral Surg Oral Med Oral Pathol Oral Radiol Endod 103: 114-119.

17. Bergenholtz $G$ (2016) Assessment of treatment failure in endodontic therapy. J Oral Rehabil 43: 753-758.

18. Koch M, Wolf E, Tegelberg A, Petersson K (2015) Effect of education intervention on the quality and long-term outcomes of root canal treatment in general practice. Int Endod J 48: 680-689.

19. Boucher Y, Toledo R (2012) Endodontie.

20. Garnier D (2000) Dictionnaire des termes de Médecine. $\left(26^{\text {th }}\right.$ edn), Paris.

21. Moller AJ, Fabricius L, Dahlen G, Sundqvist G, Happonen RP (2004) Apical periodontitis development and bacterial response to endodontic treatment. Experimental root canal infections in monkeys with selected bacterial strains. Eur $\mathrm{J}$ Oral Sci 112: 207-215.

22. Fabricius L, Dahlen G, Sundqvist G, Happonen RP, Moller AJ (2006) Influence of residual bacteria on periapical tissue healing after chemomechanical treatment and root filling of experimentally infected monkey teeth. Eur J Oral Sci 114: 278-285. 